\title{
Teleophthalmology techniques increase ophthalmic examination distance
}

\author{
Fadi R. Ghazala $\mathbb{D}^{1} \cdot$ Ruth Hamilton $\mathbb{D}^{2,3} \cdot$ Mario E. Giardini $\mathbb{C}^{4} \cdot$ lain A. T. Livingstone ${ }^{5}$
}

Received: 19 June 2020 / Revised: 2 July 2020 / Accepted: 7 July 2020 / Published online: 16 July 2020

(c) The Royal College of Ophthalmologists 2020

\section{To the Editor:}

In response to 'Quantifying examination distance in ophthalmic assessments' [1].

We read the above correspondence with interest. In ophthalmic care, proximity to the patient is often necessary. In cases where patients are non-mobile, bedside reviews may bring the ophthalmologist even closer, particularly if a portable slit lamp or direct ophthalmoscopy is required. Moreover, in the Coronavirus disease 2019 (COVID-19) pandemic, PPE including a face shield makes biomicroscopic slit lamp examination difficult as there is a physical barrier between the examiner and the oculars. The proximity required may put the ophthalmologist at increased risk of acquiring COVID-19 [2]. Social distancing has become a key concept to reduce the spread of COVID-19, with the WHO recommending keeping a $1 \mathrm{~m}$ distance to others.

Teleophthalmology has seen an increased role in service delivery in Scotland [3]. Whilst telemedicine is being used in service redesign, the added benefit with regards to COVID-19 is the increased proximity it affords during examination. Furthermore, by replacing eye-to-eye direct line-of-sight with a digital image, recording or casting visualised signs is straightforward. This allows scrubbing of video to find relevant clinical signs. In the case of paediatric imaging where interpretation is frequently based on a fleeting glimpse, the examiner can now rewind and focus on relevant frames, theoretically gleaning more information from a shorter exam. In addition, where second opinions are required from senior colleagues, the facility to record may reduce the necessity for re-examination by others, further minimising clinician-patient contacts.

We compared examination distances for standard examination techniques with those for equivalent teleophthalmology devices. An adaptor (Celestron NexYZ, Torrance, CA, USA) was used to mount a mobile device onto the oculars of a portable slit lamp. The Glasgow Retinal Imaging Adaptor model P046c-01 (Medical Devices Unit, NHS Greater Glasgow \& Clyde, UK) was used for retinal examination as a comparison to direct ophthalmoscopy. The slit lamp distance was compared to examination distance of an iPad (Apple, CA, USA) mounted to the oculars of a slit lamp using a 3D printer adaptor (Fig. 1). For each of the examinations, the mean of three repeated nose-to-nose distance calculations was compared (paired $t$-test) for the standard set-up and for the telemedicine set-up.

Slit lamp examination distance increased from 27 to $67 \mathrm{~cm}$. Portable slit lamp examination distance increased from 18 to $55 \mathrm{~cm}$. Examination distance increased from $5 \mathrm{~cm}$ for the direct ophthalmoscope to $47 \mathrm{~cm}$ for the Glasgow Retinal Imaging Adaptor (Table 1). The increased distances were not only highly statistically significant, but clinically relevant since the risk of infection reduces with distance even over the close proximities considered here [4].
Fadi R. Ghazala

fadi.ghazala@nhs.net

1 Tennent Institute of Ophthalmology, NHS Greater Glasgow and Clyde, Glasgow, UK

2 Department of Clinical Physics and Bioengineering, Royal Hospital for Children, NHS Greater Glasgow and Clyde, Glasgow, UK
College of Medical, Veterinary and Life Sciences, University of Glasgow, Glasgow, UK

4 Department of Biomedical Engineering, University of Strathclyde, Glasgow, UK

5 Forth Valley NHS Trust, Falkirk, UK 
Fig. 1 Teleophthalmology devices. a Retinal adaptor; b iPad adapted slit lamp; c adapted (NexYZ) portable slit-lamp.

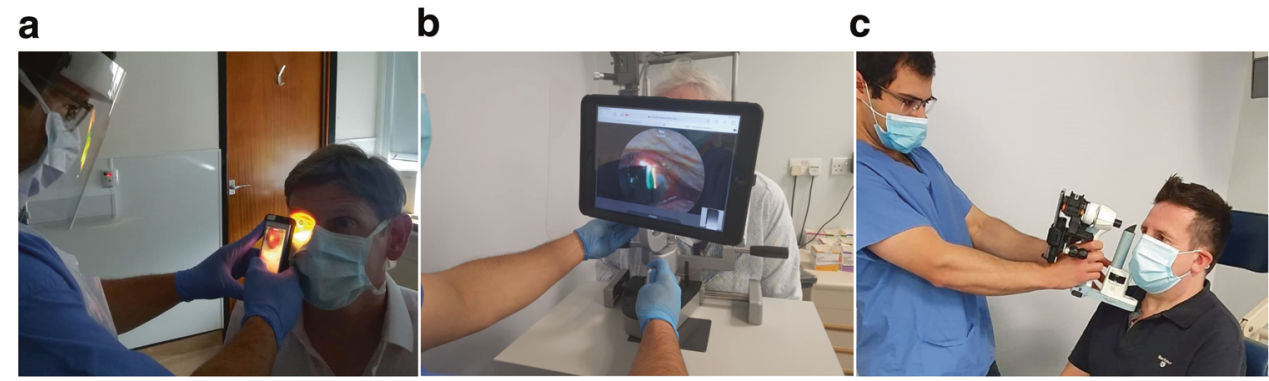

Table 1 Mean nose-to-nose measurements of various examinations.

\begin{tabular}{|c|c|c|}
\hline Examination & Nose-to-nose measurement $(\mathrm{cm})$ & Difference \\
\hline Standard slit lamp & 27 & $40 \mathrm{~cm}(95 \%$ CI $37.52-42.48, p 0.0002)$ \\
\hline Modified (iPad adapted) slit lamp & 67 & \\
\hline Portable slit lamp & 18 & $37 \mathrm{~cm}(95 \%$ CI 34.46-40.20, $p 0.0003)$ \\
\hline Modified (NexYZ) portable slit lamp & 55 & \\
\hline Direct ophthalmoscope & 5 & $42 \mathrm{~cm}(95 \%$ CI $39.46-45.20, p$ 0.0002) \\
\hline Glasgow Retinal Imaging Adaptor & 47 & \\
\hline
\end{tabular}

We conclude that teleophthalmology devices have value in reducing proximity of an ophthalmologist to a patient during examination.

Consent was obtained for the publication of all images.

Acknowledgements Department of Biomedical Engineering, University of Strathclyde, Glasgow, UK - optical design of retinal adaptor. Stephen Dacombe and Robin Sayer (Medical Devices Unit, NHS Greater Glasgow and Clyde, Glasgow, UK)—manufacture of retinal adaptor.

\section{Compliance with ethical standards}

Conflict of interest The authors declare that they have no conflict of interest.

Publisher's note Springer Nature remains neutral with regard to jurisdictional claims in published maps and institutional affiliations.

\section{References}

1. Moussa G, Mandal P, Mathews N, Lee R. Quantifying examination distance in ophthalmic assessments. Eye. 2020. https://doi.org/10. 1038/s41433-020-0978-x.

2. Kuo IC, O'Brien TP. COVID-19 and ophthalmology: an underappreciated occupational hazard. Infect Control Hosp Epidemiol. 2020. https://doi.org/10.1017/ice.2020.238.

3. NHS Scotland. National Eye Health Framework for the Coronavirus (COVID-19) Pandemic. 2020. https://communityeyecare. scot.nhs.uk/media/1044/covid19-national-eye-health-frameworkeyehealth-scotland-final.pdf. Accessed 1 June 2020.

4. Chu DK, Akl EA, Duda S, Solo K, Yaacoub S, Schünemann HJ, et al. Physical distancing, face masks, and eye protection to prevent person-to-person transmission of SARS-CoV-2 and COVID-19: a systematic review and meta-analysis. The Lancet. 2020;395:1973-87. 